\title{
Population pharmacokinetics of clozapine and its primary metabolite norclozapine in Chinese patients with schizophrenia
}

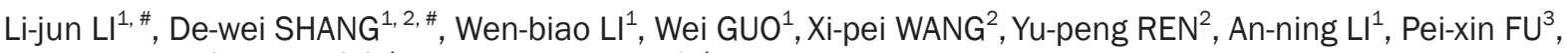 \\ Shuang-min $\mathrm{JI}^{2}$, Wei $\mathrm{LU}^{2,4, *}$, Chuan-yue WANG ${ }^{1, *}$ \\ ${ }^{1}$ Laboratory of Clinical Psychopharmacology, Beijing An Ding Hospital, Capital Medical University, Beijing 100088, China; ${ }^{2}$ Department \\ of Pharmaceutics, School of Pharmaceutical Sciences, Peking University, Beijing 100191, China; ${ }^{3}$ Judicial Expertise Center, Beijing An \\ Kang Hospital, Hancunhe Town, Beijing 102406, China; ${ }^{4}$ The State Key Laboratory of Natural and Biomimetic Drugs, Peking University, \\ Beijing 100191, China
}

Aim: To develop a combined population pharmacokinetic model (PPK) to assess the magnitude and variability of exposure to both clozapine and its primary metabolite norclozapine in Chinese patients with refractory schizophrenia via sparse sampling with a focus on the effects of covariates on the pharmacokinetic parameters.

Methods: Relevant patient concentration data (eg, demographic data, medication history, dosage regimen, time of last dose, sampling time, concentrations of clozapine and norclozapine, etc) were collected using a standardized data collection form. The demographic characteristics of the patients, including sex, age, weight, body surface area, smoking status, and information on concomitant medications as well as biochemical and hematological test results were recorded. Persons who had smoked 5 or more cigarettes per day within the last week were defined as smokers. The concentrations of clozapine and norclozapine were measured using a HPLC system equipped with a UV detector. PPK analysis was performed using NONMEM. Age, weight, sex, and smoking status were evaluated as main covariates. The model was internally validated using normalized prediction distribution errors.

Results: A total of 809 clozapine concentration data sets and 808 norclozapine concentration data sets from 162 inpatients (74 males, 88 females) at multiple mental health sites in China were included. The one-compartment pharmacokinetic model with mixture error could best describe the concentration-time profiles of clozapine and norclozapine. The population-predicted clearance of clozapine and norclozapine in female nonsmokers were 21.9 and $32.7 \mathrm{~L} / \mathrm{h}$, respectively. The population-predicted volumes of distribution for clozapine and norclozapine were 526 and $624 \mathrm{~L}$, respectively. Smoking was significantly associated with increases in the clearance (clozapine by $45 \%$; norclozapine by $54.3 \%$ ). The clearance was significantly greater in males than in females (clozapine by $20.8 \%$; norclozapine by $24.2 \%$ ). The clearance of clozapine and norclozapine did not differ significantly between Chinese patients and American patients.

Conclusion: Smoking and male were significantly associated with a lower exposure to clozapine and norclozapine due to higher clearance. This model can be used in individualized drug dosing and therapeutic drug monitoring.

Keywords: schizophrenia; clozapine; norclozapine; population pharmacokinetics; NONMEM; smoking; male; individualized drug dosing; therapeutic drug monitoring

Acta Pharmacologica Sinica (2012) 33: 1409-1416; doi: 10.1038/aps.2012.71; published online 23 Jul 2012

\section{Introduction}

Clozapine is a tricyclic dibenzodiazepine antipsychotic drug that is commonly used in the treatment of schizophrenia, particularly in patients who are refractory or intolerant to the

\footnotetext{
"These authors contributed equally to this paper.

* To whom correspondence should be addressed.

E-mail wang.cy@163.net (Chuan-yue WANG);

luwei_pk@bjmu.edu.cn (Wei LU)

Received 2012-03-12 Accepted 2012-05-11
}

side effects of traditional antipsychotics ${ }^{[1]}$. Although clozapine may cause agranulocytosis in some patients, the incidence is approximately $0.38 \%$. In addition, clozapine does not cause acute extrapyramidal toxicity or irreversible neurologic side effects $^{[2]}$. Because its benefits outweigh its side effects, clozapine has been accepted internationally as antipsychotic drug and is used to treat approximately $31.7 \%$ of Chinese schizophrenia patients ${ }^{[3]}$. Clozapine is metabolized primarily by human cytochrome P450 (CYP) isozyme 1A2, yielding a 
pharmacologically active metabolite, norclozapine ${ }^{[4]}$. Several investigators have shown that the degree to which clozapine is converted into norclozapine predicts the clinical outcome with respect to multiple measures of cognition, negative and positive symptoms, as well as quality of life $\mathrm{e}^{[5,6]}$. Thus, routine therapeutic drug monitoring (TDM) of clozapine and norclozapine is recommended to ensure safety and minimize toxic adverse events ${ }^{[7]}$.

Significant intra- and inter-individual pharmacokinetic (PK) variability for clozapine and norclozapine has been observed in routine $\mathrm{TDM}^{[8,9]}$. The influences of sex, smoking, CYP1A2 activity and other factors on clozapine plasma concentrations have been previously reported ${ }^{[10-13]}$. However, studies exploring the effect of sex and smoking status on dose and concentration have shown mixed effects, and the clinical administration of clozapine is characterized by frequent dose changes and different dose intervals. Therefore, comparing only dosenormalized concentrations does not ensure the magnitude of the effect or provide suggestions for suitable personalized therapy.

Population pharmacokinetic (PPK) analysis is a robust tool for obtaining valuable PK information from both sparsely and intensively sampled data. The influence of potential covariates on drug exposure can also be quantitatively evaluated in PPK analysis by incorporating these covariates into the modeling process. This method also enables the characterization of both the inter-individual and intra-individual variabilities. Our research group has built a PPK model for clozapine in Chinese patients using retrospective TDM data ${ }^{[14]}$. However, this model was limited by the evaluation methods and the small sample size and failed to provide thorough information on the PPK parameters of both clozapine and norclozapine. A review of the recent literature yielded two studies by one group on the PPK analysis of clozapine and norclozapine simultaneously. Robert R BIES et al developed a combined PPK model of clozapine and norclozapine for American patients based on the assumption that all clozapine was excreted as norclozapine, and they estimated the PK parameters using a fixed central volume of $7 \mathrm{~L} / \mathrm{kg}$ for both clozapine and norclozapine ${ }^{[11,15]}$ using retrospective TDM data and outpatient concentration data.

In this study, another structural model for simultaneously analyzing clozapine and norclozapine in Chinese patients with schizophrenia was built without a fixed volume of distribution. The concentration data were collected using a prospective study design covering plasma data for both the absorption and distribution phases to calculate the PK parameters for clozapine and norclozapine. The results of this study may be used to better understand the PK characteristics of clozapine in Chinese patients with schizophrenia. Ultimately, our goal is to link the PK model with the pharmacodynamics (PD) of clozapine's antipsychotic effect to guide personalized therapy.

\section{Materials and methods} Patients and data collection

The inclusion criteria for patients included a diagnosis of schizophrenia according to the Diagnostic and Statistical Manual of Mental Disorders IV (DSM-IV) established by administering the Structural Clinical Interview for DSM-IV (SCID) augmented by a review of the medical records. Most patients were treated with oral clozapine twice or three times per day. In this study, only one brand of clozapine (Jiangsu Nhwa pharmaceutical corporation limited, Xuzhou, China) was administered. Persons who had smoked five or more cigarettes per day within the last week were defined as smokers. Compliance was assessed by the determination of the serum concentration of clozapine several times during the study and by interview with the attending psychiatrists. Twenty percent of total samples were obtained between $0.5 \mathrm{~h}$ and $4.5 \mathrm{~h}$ after the last dose, and the remaining samples were obtained between $8.5 \mathrm{~h}$ and $15.5 \mathrm{~h}$ after the last dose. Previous institutional ethical approval was obtained, and all patients in the study gave written informed consent.

Relevant patient concentration data (eg, demographic data, medication history, dosage regimen, time of last dose, sampling time, concentrations of clozapine and norclozapine, etc) were collected using a standardized data collection form. The demographic characteristics of the patients, including sex, age, weight, body surface area, smoking status, and information on concomitant medications as well as biochemical and hematological test results were recorded.

\section{Determination of clozapine and norclozapine concentrations in serum}

An Agilent 1200 integrated high-performance liquid chromatography system equipped with a UV detector was used. The separation of the compounds was performed on an Inertsil ODS-3 column ( $5 \mu \mathrm{m}, 150 \mathrm{~mm} \times 4.6 \mathrm{~mm}$, id $)$ at room temperature. The column was preceded by a Phenomenex SecurityGuard ${ }^{\mathrm{TM}} \mathrm{C} 18$ guard column $(4.0 \mathrm{~mm} \times 3.0 \mathrm{~mm}$ id $)$. The mobile phase consisted of methanol:acetonitrile:water: acetic acid:butylamine at 32:73:95:2:1 $(v / v / v / v)$ and was pumped at a flow-rate of $1.0 \mathrm{~mL} / \mathrm{min}$. Detection was performed at the wavelength of $254 \mathrm{~nm}$. The height ratios of the compounds' peaks to the desipramine peak (internal standard) were employed for all calculations. The lower limits of detection for clozapine and norclozapine were both $0.08 \mu \mathrm{mol} / \mathrm{L}$. The coefficient of variation was less than $5 \%$ for both clozapine and norclozapine ${ }^{[16]}$.

\section{Population pharmacokinetic modeling}

The PPK analysis was performed using NONMEM (version 7, level 1.0, ICON Development Solutions, Ellicott City, MD, USA $)^{[17-19]}$. The plasma concentration-time profiles for clozapine and norclozapine were described by a base structural model using the subroutine ADVAN5 $5^{[20]}$. The structural model (Figure 1) was based on the following assumptions: a proportion of clozapine was converted into norclozapine in the central compartment of clozapine through a first-order conversion mechanism and the rest was converted into other metabolites. The PK structural model was parameterized in terms of the apparent clearance and the apparent distribution 


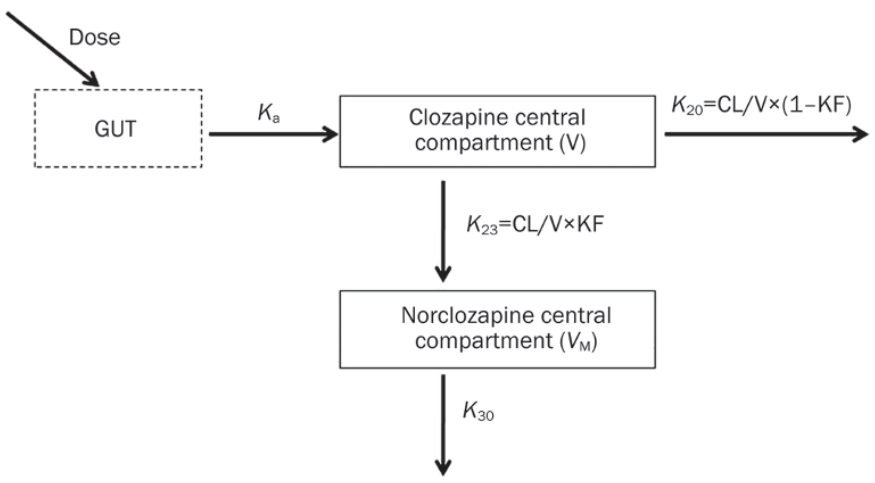

Figure 1. Population pharmacokinetic model structure for clozapine and norclozapine. $K_{20}$, elimination rate constant for clozapine; $\mathrm{V}$, volume of distribution for clozapine; $K_{23}$, rate constant for the conversion of clozapine into norclozapine; $K_{30}$, elimination rate constant for norclozapine; $V_{\mathrm{M}}$, volume of distribution for norclozapine; $\mathrm{KF}$, fraction of clozapine converted into norclozapine.

volume of clozapine (CL/F and V/F), where $\mathrm{F}$ is the unknown oral bioavailability of clozapine; the clearance and the distribution volume of norclozapine $\left(\mathrm{CL}_{\mathrm{M}}\right.$ and $\left.V_{\mathrm{M}}\right)$; and the fraction of the absorbed dose of clozapine converted into norclozapine (KF). A Bayesian approach conditioned on the population characteristics was used to estimate individual specific parameters. First-order conditional estimation methods (FOCE) and first-order conditional estimation methods with interaction (FOCE-I) were tested during model development.

The unexplained random variability in the individual values of the structural model parameters were described in the interindividual variability (IIV) model. The IIV of the PK parameters was assumed to be log normally distributed; the relationship between a parameter and its variance could be expressed as follows:

$$
P_{\mathrm{j}}=P_{\mathrm{TV}} \times e^{\mathrm{np}}
$$

where $P_{\mathrm{j}}$ is the value of the parameter as predicted for the individual $\mathrm{j}, P_{T V}$ is the population typical value of the parameter, and $\eta_{\mathrm{p}}$ represents the difference in the estimated parameter for the jth subject from the population typical value, which was identically distributed with a mean of zero and a variance of $\omega_{\mathrm{p}}^{2}$.

The residual error was tested using additive, proportional, and combined error structures as follows:

Additive error model: $y_{\mathrm{ij}}=y_{\mathrm{ij}}{ }^{\prime}+\varepsilon_{\mathrm{ij}}$

Proportional error model: $y_{\mathrm{ij}}=y_{\mathrm{ij}}{ }^{\prime} \times\left(1+\varepsilon_{\mathrm{ij}}\right)$

Combined additive and proportional error model:

$$
y_{\mathrm{ij}}=y_{\mathrm{ij}}{ }^{\prime} \times\left(1+\varepsilon_{\mathrm{ij}}\right)+\varepsilon_{\mathrm{ij}}{ }^{\prime}
$$

where $y_{\mathrm{ij}}$ is the jth observation in the ith individual; $y_{\mathrm{ij}}{ }^{\prime}$ is the model's predicted value; and $\varepsilon_{\mathrm{ij}}$ and $\varepsilon_{\mathrm{ij}}{ }^{\prime}$ are the normally distributed random errors with mean values of zero and variances of $\sigma_{1}{ }^{2}$ and $\sigma_{2}{ }^{2}$, respectively. The residual error model was tested for the parent and metabolite in this model.

The full model was built by stepwise forward inclusion and the effect of each covariate was evaluated. The individual PK parameters obtained from the basic model were plotted against each covariate separately and the scatter plots helped to identify the trends and the regression pattern. Then, each covariate was incorporated stepwise into the basic regression model to develop the intermediate and full models. When the addition of a covariate resulted in a decrease in the objective function value $(\mathrm{OFV})$ of $>6.63$ (chi-square, $P<0.01, \mathrm{df}=1$ ), the covariate was considered statistically significant during the covariate forward-inclusion process. In this study, both continuous covariates (eg, age and weight) and discrete covariates (eg, sex, smoking status, and concomitant medications) were introduced into each parameter in a stepwise fashion. The relationship between the population typical value of the clearance and a continuous covariate such as age was evaluated using the following relationship:

$$
\text { TVCL }=\theta_{\mathrm{CLF}} \times\left(1+\left(\mathrm{AGE}-\mathrm{AGE}_{\mathrm{AVE}}\right) \times \theta_{\mathrm{AGE}}\right)
$$

where TVCL is the population typical value of clearance, AGE is the age of the individual in years, $\mathrm{AGE}_{\mathrm{AVE}}$ is the average value of the age in the population and $\theta_{\mathrm{AGE}}$ is the factor contributed by the covariate.

The following example shows the effect of a discrete covariate such as sex on clearance $(\mathrm{CL} / \mathrm{F})$ :

$$
\text { TVCL }=\theta_{\mathrm{CLF}} \times\left(1+\mathrm{SEX} \times \theta_{\mathrm{SEX}}\right)
$$

When sex is female (female $=0$, male $=1$ ), TVCL equals $\theta_{\mathrm{CL}}$. For male subjects, the $\theta_{\text {cov }}$ term is added to the population estimate of clearance to modify this estimate.

For the final model, a backward elimination process was employed to identify significant covariates. The covariates in the full model were excluded one by one. The OFV was compared with that of the full model. A covariate was retained in the model when the elimination of that covariate resulted in an increase in the OFV of 7.88 (chi-square, $P<0.005, \mathrm{df}=1$ ). We selected the model according to the reduction in the OFV value, goodness-of-fit plots, reductions in the IIV of structure model parameters, residual error, robust model parameter estimation, and model stability.

\section{Model validation}

Because the data were collected from routine TDM, there was a wide range in the time points, the number of drug administrations, and the drug dose for different patients. Additionally, observations were sparse data. To evaluate a model on the basis of such a complicated data set, the normalized prediction distribution error (NPDE) method, recently developed by Brendel and colleagues, was suitable. NPDE is a type of model evaluation method that can be used for internal or external evaluation. The NPDE should follow the normal standard distribution in theory, such that it can be used to evaluate different types of study designs ${ }^{[21]}$. This method was implemented using the NPDE add-on software package, which was run in $\mathrm{R}$ (version 2.12.2).

\section{Results}

A total of 809 clozapine concentration data sets and 808 norclozapine concentration data sets were collected from 162 patients (74 males, 88 females) at multiple mental health sites in China. The characteristics of the patients are summarized 
in Table 1.

Table 1. Demographic characteristics of patients.

\begin{tabular}{|c|c|}
\hline Characteristic & All Patients \\
\hline Number of patients & 162 \\
\hline Gender (male/female) & $74 / 88$ \\
\hline Number of concentration data & $1617(809 / 808)$ \\
\hline \multicolumn{2}{|l|}{ (Clozapine/Norclozapine) } \\
\hline Age (mean $\pm S D$, years) (range) & $35.5 \pm 10.6(18-59)$ \\
\hline \multicolumn{2}{|l|}{ Number of the patients smoking } \\
\hline Female nonsmoker & 72 \\
\hline Female smoker & 2 \\
\hline Male nonsmoker & 40 \\
\hline Male smoker & 48 \\
\hline Clozapine concentration, mean \pm SD $\mu \mathrm{mol} / \mathrm{L}$ & $1.14 \pm 0.73$ \\
\hline Norclozapine concentration, mean \pm SD $\mu \mathrm{mol} / \mathrm{L}$ & $0.54 \pm 0.32$ \\
\hline
\end{tabular}

A one-compartment pharmacokinetic model with mixture error best described the concentration of clozapine and norclozapine. The first-order absorption rate constant $\left(K_{a}\right)$ for clozapine was fixed at $1.3 \mathrm{~h}^{-1}$ based on several pharmacokinetic studies that obtained rich data describing the pharmacokinetics of clozapine in patients ${ }^{[12]}$. The fraction of the absorbed dose of clozapine converted into norclozapine (KF) was fixed at 0.66 in published papers and was validated by the ratio of the mean amount of norclozapine to the mean amount of clozapine at steady-state in these articles ${ }^{[22,23]}$. The PK parameters determined using the FOCE-I method deviated more from the theoretical population and individual predictions than the parameters determined using the FOCE. Therefore, the FOCE method was selected for the model described. The goodnessof-fit plots for clozapine and norclozapine were good (Figure 2).

In the final model, sex, and smoking status were identified as significant covariates for the clearance of clozapine and norclozapine. Adding each covariate independently using stepwise forward inclusion improved the fit of the model. The population-predicted clearance of clozapine and norclozapine in female nonsmokers were 21.9 and $32.7 \mathrm{~L} / \mathrm{h}$, and the population-predicted volumes of distribution for clozapine and norclozapine were 526 and $624 \mathrm{~L}$, respectively. Smoking was associated with increases in the clearance of clozapine and norclozapine of $45 \%(P<0.001)$ and $54.3 \%(P<0.001)$, respectively. The clearance of clozapine and norclozapine were $20.8 \%(P<0.005)$ and $24.2 \%(P<0.005)$ greater, respectively, in males than in females (Figure 3). Other covariates such as weight and age did not significantly influence the PK parameters of clozapine and norclozapine. The combined effects of sex $(P<0.005)$ and smoking $(P<0.001)$ on the clearance of clozapine and norclozapine were determined. Male smokers were exposed to larger dosages due to the higher clearance of clozapine. The final population PK parameters are summarized in Table 2 .
Table 2. Population pharmacokinetic parameter estimates of clozapine and norclozapine.

\begin{tabular}{|c|c|c|c|}
\hline Parameters & $\begin{array}{l}\text { Parameter } \\
\text { estimates }\end{array}$ & RSE\% & $\begin{array}{l}\text { Interindividual } \\
\text { variability\% }\end{array}$ \\
\hline \multicolumn{4}{|l|}{ Clozapine } \\
\hline $\mathrm{CL} / \mathrm{F}, \mathrm{L} / \mathrm{h}$ & 21.9 & 6 & 42.9 \\
\hline esmoking & 0.45 & 34.9 & \\
\hline$\theta$ Gender & 0.208 & 44.6 & \\
\hline $\mathrm{V} / \mathrm{F}, \mathrm{L}$ & 526 & 10 & 65.7 \\
\hline$K_{a}($ Fixed), $1 / h$ & 1.3 & NA & NA \\
\hline KF (Fixed) & 0.66 & NA & NA \\
\hline \multicolumn{4}{|l|}{ Norclozapine } \\
\hline $\mathrm{CL}_{\mathrm{M}}, \mathrm{L} / \mathrm{h}$ & 32.7 & 5.6 & 42.1 \\
\hline OSmoking & 0.543 & 35.7 & \\
\hline$\theta$ Gender & 0.242 & 49.2 & \\
\hline \multirow[t]{2}{*}{$V_{M}, L$} & 624 & 5.5 & 75.6 \\
\hline & & & Residual Variability \\
\hline$\sigma_{1}(\mu \mathrm{mol} / \mathrm{L})$ & & & $S D=0.162$ \\
\hline$\sigma_{2} \%$ & & & $C V=26.6$ \\
\hline$\sigma_{3}(\mu \mathrm{mol} / \mathrm{L})$ & & & $S D=0.117$ \\
\hline$\sigma_{4} \%$ & & & $C V=16.9$ \\
\hline
\end{tabular}

$\mathrm{CL}$, clozapine clearance; $\mathrm{V}$, clozapine volume of distribution; CLM, norclozapine clearance; $V_{\mathrm{M}}$, norclozapine volume of distribution; $\mathrm{KF}$, the fraction of clozapine converting to norclozapin; $\theta$ Smoking, effect of smoking status on clearance; $\theta$ Gender, effect of smoking status on clearance; $\sigma_{1}$, coefficient of variation of additive residual error; $\sigma_{2}$, coefficient of variation of proportional residual error; N/A: not available

The graphs of clozapine (A) and norclozapine (B) for the NPDE validated the prediction of the model (Figure 4). The upper left graph is a quantile-quantile plot (QQ plot) comparing the distribution of the NPDE to the theoretical $N(0,1)$ distribution, and the upper right graph is a histogram of the NPDE with the density of $N(0,1)$ overlaid. In the two lower graphs, the NPDE is plotted against time (the independent variable $\mathrm{X}$ ) and the predicted concentration (predicted $\mathrm{Y}$ ), respectively ${ }^{[21,24,25]}$. For clozapine and norclozapine, the mean of the distribution of the NPDE was close to 0, and the variance was small. The two lower graphs show that the same trend was observed for both NPDE and the real data. These results indicate that the model of the clozapine and norclozapine concentrations was relatively accurate and reliable.

\section{Discussion}

The NONMEM method plays an important role in routine therapeutic drug monitoring, and an increasing number of researchers are using modeling to estimate PK parameters with the sparse sampling design ${ }^{[26]}$. In our study, we collected blood samples during the absorption and distribution phases and combined these samples with those for elimination phase from TDM to obtain typical values for the clearance and volume of distribution of clozapine and norclozapine. The combined PPK model was used to quantify the effects of sex and smoking status on the clearance of clozapine and norclozapine 

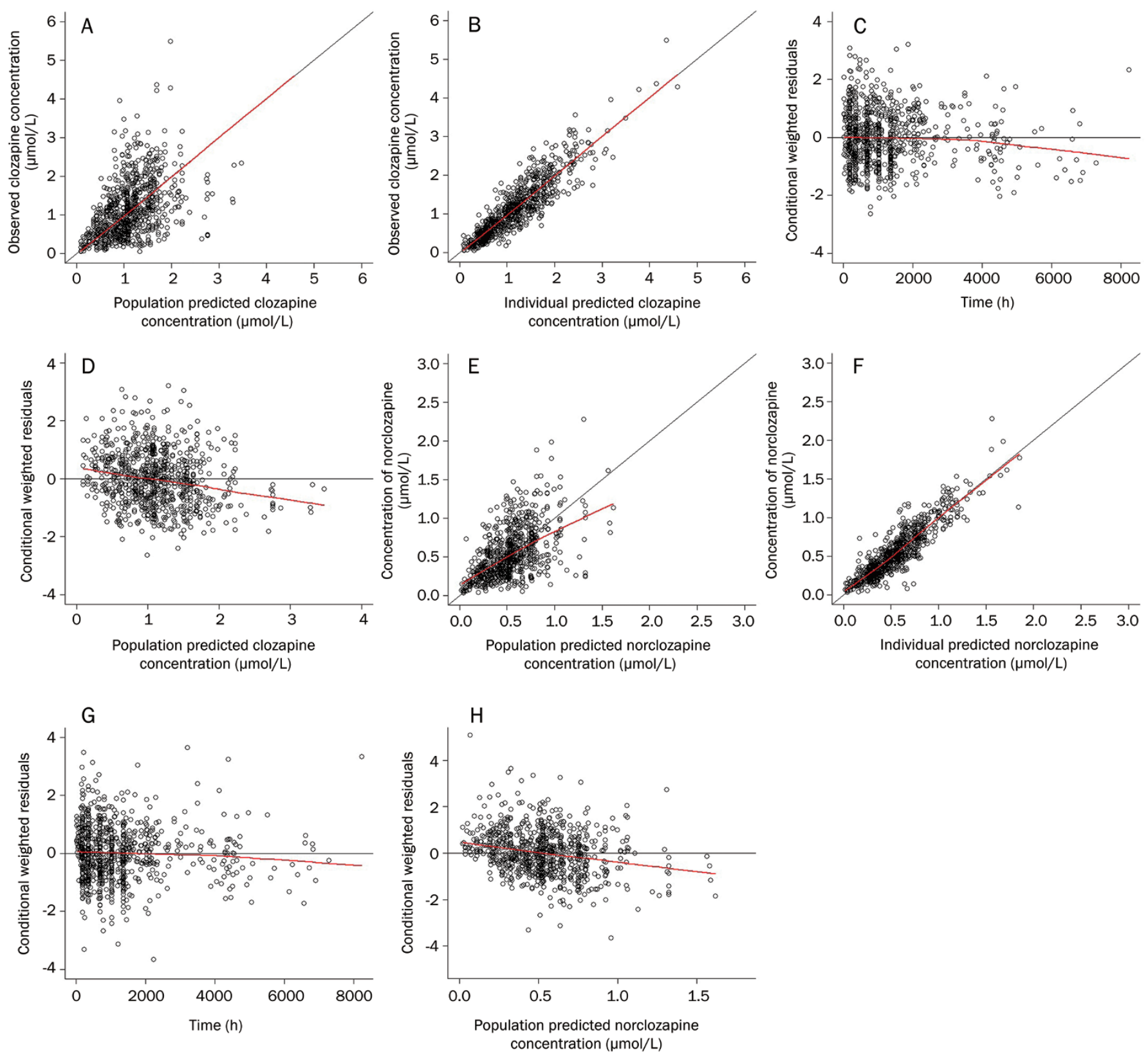

Figure 2. Diagnostic plots for the final pharmacokinetic model. Plot of the observed concentrations vs the population-predicted clozapine (A) and norclozapine (E) concentrations. Plot of the observed concentrations vs the individual population-predicted clozapine (B) and norclozapine (F) concentrations. Plot of the conditional weighted residual error (CWRES) vs the population-predicted clozapine (C) and norclozapine (G) concentrations. Plot of the conditional weighted residual error (CWRES) vs the time after first dose of clozapine (D) and norclozapine (H).
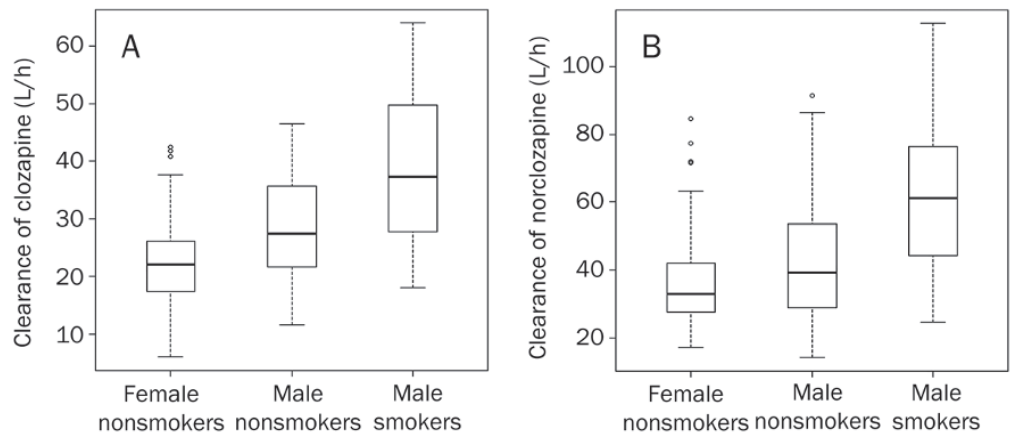

Figure 3. Boxplot with the median and interquartile range of the clearance of clozapine and norclozapine according to sex and smoking status. Clozapine clearance in female nonsmokers, male nonsmokers and male smokers (A). Norclozapine clearance in female nonsmokers, male nonsmokers, and male smokers (B). 
A Q-Q plot versus $\mathrm{N}(0,1)$ for NPDE
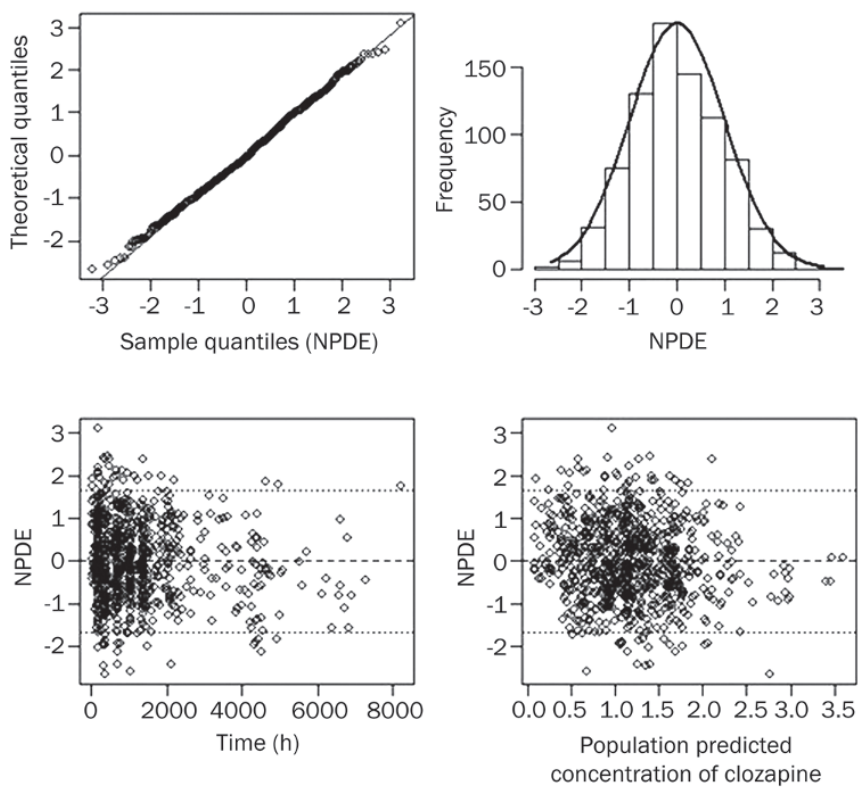

B Q-Q plot versus $\mathrm{N}(0,1)$ for NPDE
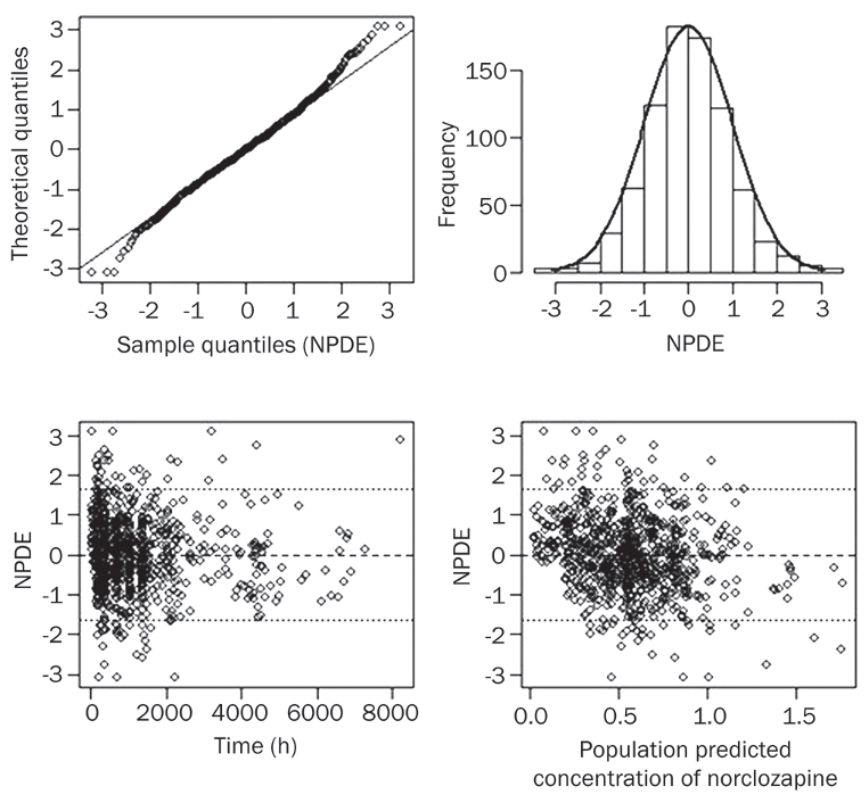

Figure 4. Results of the NPDE analysis for clozapine (A) and norclozapine (B). The left upper plot is a QQ-plot for NPDE; the right upper plot is a histogram of the NPDE; the left lower and right lower plots represent the NPDE versus time and the NPDE versus the predicted concentrations of clozapine or norclozapine, respectively.

and to predict the concentrations of these compounds at different dosage levels in Chinese patients with schizophrenia.

Clozapine can be converted into more than 10 metabolites. The primary enzymes involved in the biotransformation of clozapine are CYP1A2 and CYP3A4, which convert clozapine into norclozapine and clozapine-N-oxide, respectively. CYP2C19 may also have significant effects on the conversion of clozapine into norclozapine. CYP2C9 and CYP2D6 appear to play minor roles. Norclozapine is further converted into a more polar compound for elimination. The clozapine and norclozapine metabolic pathway had been described in detail by Dain and Khan et al ${ }^{[27,28]}$. We built a combined structural model to explain the conversion of clozapine into norclozapine (Figure 1) based on the metabolism of clozapine. Therefore, in the current study, we assumed that clozapine was partly converted into norclozapine with a conversion fraction (KF), which was more reasonable than the assumption in previous PPK studies with respect to the process of clozapine metabo$\operatorname{lism}^{[11,15,29]}$. The model proposed in this study provided an adequate fit to the data for both clozapine and norclozapine.

Smoking status was previously identified as a statistically significant covariate affecting the clearance of clozapine and norclozapine ${ }^{[30,31]}$. Because the activity of CYP1A2 is greater in smokers than in non-smokers ${ }^{[9]}$, the clearance of clozapine is likely influenced by smoking status. It was reported that smoking can increase the clearance of clozapine and that the sudden cessation of smoking can cause a significant decrease in caffeine clearance $(P<0.01)$ of $36.1 \%^{[32-34]}$. Sex was also reported to be a significant covariate affecting the clearance of clozapine and norclozapine ${ }^{[11,35,36]}$. Previous studies have shown that CYP1A2 activity was lower in females than in males ${ }^{[37]}$, whereas other studies found no differences in CYP1A2 activity between males and females and insisted that smoking could induce CYP1A2 activity and reduce clozapine clearance $^{[33,38]}$. Smoking and male were associated with lower exposure to clozapine and norclozapine due to their higher clearance. Considering the mixed effect of smoking and sex, we made use of the PPK model to quantify the mixture effect on the clearance and concentrations of clozapine and norclozapine. Although our study contained only a few female patients who smoked $(n=2)$, the effect of smoking on females could be inferred by the built model. The clearance of clozapine and norclozapine was $23.6 \%$ higher in female smokers than in male nonsmokers.

The clearance of clozapine and norclozapine in Chinese patients was similar to the published value for American patients. The results suggest that the enzyme activity in different populations is not significantly different.

The impact of age on the clearance of clozapine and norclozapine has been investigated. Haring et al ${ }^{[34]}$ found that clozapine concentrations in patients aged between 45 and 54 years were higher than that in patients aged between 18 and 26 years, but the effect was not statistically significant. Another recent study without smoking information demonstrated there was a negative effect of age on the clearance of clozapine $^{[15]}$. However, in some studies with smoking information, the investigators were unable to find a significant effect of age on the clozapine blood concentration ${ }^{[39,40]}$. In our research, the effect of age on the clearance of clozapine and norclozapine was also not statistically significant compared with the effects of smoking status and sex.

The model used in the present study was internally validated using the NPDE. The prediction for clozapine was accurate and reliable, although slight bias existed in the prediction 
for norclozapine.

There were some limitations in our study. First, smoking status was assessed using patient self-reporting and nurse evaluations. We dichotomized patients into smokers and nonsmokers but did not assess the magnitude of smoking. A lack of objective biological measures (such as the serum nicotine level) may have resulted in false-positive and falsenegative cases. Second, the intake of concomitant medications in our patients was complicated. Although these concomitant medications might have interacted with the pharmacokinetics of clozapine and norclozapine, this interaction was not adequately confirmed by our study design.

In conclusion, a one-compartment model with first-order absorption adequately described the concentration data for clozapine and its active metabolite norclozapine. Smoking status and sex were identified as two significant covariates affecting the clearance of clozapine and norclozapine. These findings may account for some of the variability in clozapine and norclozapine exposure, and the dosage regimen in Chinese populations may need to be adjusted to improve the efficacy and safety of clozapine based on patients' smoking status and sex.

\section{Acknowledgements}

The authors are extremely grateful to Chen-hui DENG, Liang ZHANG and Fu-chun ZHOU, who provided valuable comments.

This study was supported by the National Natural Science Foundation of China (Grant № 30770776), the National High Technology Research and Development Program of China (863 Program) (Grant No 2009AA022702), the Beijing Municipal Education Commission Science and Technology Development Program (Grant No KM201110025025), the Beijing Municipal Science and Technology Commission (Grant № D101107047810001) and the Beijing Postdoctoral Research Foundation (Grant No 2011ZZ-11).

\section{Author contribution}

Chuan-yue WANG and Wei LU designed the research; Chuan-yue WANG, Li-jun LI, Pei-xin FU, and An-ning LI performed the research; Wen-biao LI and Wei GUO determined the serum concentrations of clozapine and norclozapine; Wei LU, De-wei SHANG, Li-jun LI, Xi-pei WANG, Yu-peng REN, and Shuang-min JI built the model; and De-wei SHANG and Li-jun LI wrote the paper.

\section{References}

1 Kane J, Honigfeld G, Singer J, Meltzer H. Clozapine for the treatment-resistant schizophrenic. A double-blind comparison with chlorpromazine. Arch Gen Psychiatry 1988; 45: 789-96.

2 Lieberman JA. Maximizing clozapine therapy: managing side effects. J Clin Psychiatry 1998; 59: 38-43.

3 Si TM, Shu L, Yu X, Ma C, Wang GH, Bai PS, et al. The second crosssectional study on antipsychotic drug patterns of schizophrenia in China. Chin J Psychiatry 2010; 43: 31-6.

4 Bertilsson L, Carrillo JA, Dahl ML, Llerena A, Alm C, Bondesson U, et al. Clozapine disposition covaries with CYP1A2 activity determined by a caffeine test. Br J Clin Pharmacol 1994; 38: 471-3.

5 Flanagan RJ, Yusufi B, Barnes TR. Comparability of whole-blood and plasma clozapine and norclozapine concentrations. Br J Clin Pharmacol 2003; 56: 135-8.

6 Mauri MC, Volonteri LS, Dell'Osso B, Regispani F, Papa P, Baldi M, et al. Predictors of clinical outcome in schizophrenic patients responding to clozapine. J Clin Psychopharmacol. 2003; 23: 660-4.

7 Couchman L, Morgan PE, Spencer EP, Flanagan RJ. Plasma clozapine, norclozapine, and the clozapine:norclozapine ratio in relation to prescribed dose and other factors: data from a therapeutic drug monitoring service, 1993-2007. Ther Drug Monit 2010; 32: 438-47.

8 Diaz FJ, de Leon J, Josiassen RC, Cooper TB, Simpson GM. Plasma clozapine concentration coefficients of variation in a long-term study. Schizophr Res 2005; 72: 131-5.

9 Doude van Troostwijk LJ, Koopmans RP, Vermeulen HD, Guchelaar HJ. CYP1A2 activity is an important determinant of clozapine dosage in schizophrenic patients. Eur J Pharm Sci 2003; 20: 451-7.

10 Rostami-Hodjegan A, Amin AM, Spencer EP, Lennard MS, Tucker GT, Flanagan RJ. Influence of dose, cigarette smoking, age, sex, and metabolic activity on plasma clozapine concentrations: a predictive model and nomograms to aid clozapine dose adjustment and to assess compliance in individual patients. J Clin Psychopharmacol 2004; 24: $70-8$.

11 Ng W, Uchida H, Ismail Z, Mamo DC, Rajji TK, Remington G, et al. Clozapine exposure and the impact of smoking and gender: a population pharmacokinetic study. Ther Drug Monit 2009; 31: 360-6.

12 Jerling M, Merle Y, Mentre F, Mallet A. Population pharmacokinetics of clozapine evaluated with the nonparametric maximum likelihood method. Br J Clin Pharmacol 1997; 44: 447-53.

13 Dailly E, Urien S, Chanut E, Claudel B, Guerra N, Femandez C, et al. Evidence from a population pharmacokinetics analysis for a major effect of CYP1A2 activity on inter- and intraindividual variations of clozapine clearance. Prog Neuropsychopharmacol Biol Psychiatry 2002; 26: 699-703.

14 Qiu XW, Fu PX, Wang CY, Liu M, Zhou TY, Lu W. Population pharmacokinetics research of clozapine in Chinese schizophrenic patients. Yao Xue Xue Bao 2009; 44: 785-92.

15 Ismail Z, Wessels AM, Uchida H, Ng W, Mamo DC, Rajji TK, et al. Age and sex impact clozapine plasma concentrations in inpatients and outpatients with schizophrenia. Am J Geriatr Psychiatry 2012; 20: 53-60.

16 Volpicelli SA, Centorrino F, Puopolo PR, Kando J, Frankenburg FR, Baldessarini RJ, et al. Determination of clozapine, norclozapine, and clozapine-N-oxide in serum by liquid chromatography. Clin Chem 1993; 39: 1656-9.

17 Sheiner LB, Beal SL. Evaluation of methods for estimating population pharmacokinetics parameters. I. Michaelis-Menten model: routine clinical pharmacokinetic data. J Pharmacokinet Biopharm 1980; 8: 553-71.

18 Sheiner BL, Beal SL. Evaluation of methods for estimating population pharmacokinetic parameters. II. Biexponential model and experimental pharmacokinetic data. J Pharmacokinet Biopharm 1981; 9: 635-51.

19 Sheiner LB, Beal SL. Evaluation of methods for estimating population pharmacokinetic parameters. III. Monoexponential model: routine clinical pharmacokinetic data. J Pharmacokinet Biopharm 1983; 11: 303-19.

20 Feng Y, Pollock BG, Coley K, Marder S, Miller D, Kirshner M, et al. Population pharmacokinetic analysis for risperidone using highly sparse sampling measurements from the CATIE study. $\mathrm{Br} J$ Clin Pharmacol 2008; 66: 629-39. 
21 Comets E, Brendel K, Mentre F. Computing normalised prediction distribution errors to evaluate nonlinear mixed-effect models: the npde add-on package for R. Comput Methods Programs Biomed 2008; 90 : 154-66.

22 Golden G, Honigfeld G. Bioequivalence of clozapine orally disintegrating 100-mg tablets compared with clozapine solid oral 100-mg tablets after multiple doses in patients with schizophrenia. Clin Drug Investig 2008; 28: 231-9.

23 Lin SK, Chang WH, Chung MC, Lam YW, Jann MW. Disposition of clozapine and desmethylclozapine in schizophrenic patients. J Clin Pharmacol 1994; 34: 318-24.

24 Krekels EH, van Hasselt JG, Tibboel D, Danhof M, Knibbe CA. Systematic evaluation of the descriptive and predictive performance of paediatric morphine population models. Pharm Res 2011; 28 : 797-811.

25 Knibbe CA, Krekels EH, van den Anker JN, DeJongh J, Santen GW, van Dijk M, et al. Morphine glucuronidation in preterm neonates, infants and children younger than 3 years. Clin Pharmacokinet 2009; 48: 371-85.

26 Ette El, Williams PJ. Population pharmacokinetics I: background, concepts, and models. Ann Pharmacother 2004; 38: 1702-6.

27 Dain JG, Nicoletti J, Ballard F. Biotransformation of clozapine in humans. Drug Metab Dispos 1997; 25: 603-9.

28 Khan AY, Preskorn SH. Examining concentration-dependent toxicity of clozapine: role of therapeutic drug monitoring. J Psychiatr Pract 2005; 11: 289-301.

29 Guitton C, Kinowski JM, Gomeni R, Bressolle F. A kinetic model for simultaneous fit of clozapine and norclozapine concentrations in chronic schizophrenic patients during long-term treatment. Clin Drug Investig 1998; 16: 35-43.

30 Lee ST, Ryu S, Nam HJ, Lee SY, Hong KS. Determination of pharmacokinetic properties of clozapine and norclozapine in Korean schizophrenia patients. Int Clin Psychopharmacol 2009; 24: 139-44.

31 Gaertner I, Gaertner HJ, Vonthein R, Dietz K. Therapeutic drug moni- toring of clozapine in relapse prevention: a five-year prospective study. J Clin Psychopharmacol 2001; 21: 305-10.

32 Faber MS, Fuhr U. Time response of cytochrome P450 $1 \mathrm{~A} 2$ activity on cessation of heavy smoking. Clin Pharmacol Ther 2004; 76: 178-84.

33 van der Weide J, Steijns LS, van Weelden MJ. The effect of smoking and cytochrome P450 CYP1A2 genetic polymorphism on clozapine clearance and dose requirement. Pharmacogenetics 2003; 13: 16972.

34 Haring C, Meise U, Humpel C, Saria A, Fleischhacker WW, Hinterhuber $\mathrm{H}$. Dose-related plasma levels of clozapine: influence of smoking behaviour, sex and age. Psychopharmacology (Berl) 1989; 99: S38-40.

35 Wu RR, Zhao JP, Zhai JG, Guo XF, Guo WB. Sex difference in effects of typical and atypical antipsychotics on glucose-insulin homeostasis and lipid metabolism in first-episode schizophrenia. J Clin Psychopharmacol 2007; 27: 374-9.

36 Palego L, Biondi L, Giannaccini G, Sarno N, Elmi S, Ciapparelli A, et al. Clozapine, norclozapine plasma levels, their sum and ratio in 50 psychotic patients: influence of patient-related variables. Prog Neuropsychopharmacol Biol Psychiatry 2002; 26: 473-80.

37 Relling MV, Lin JS, Ayers GD, Evans WE. Racial and gender differences in N-acetyltransferase, xanthine oxidase, and CYP1A2 activities. Clin Pharmacol Ther 1992; 52: 643-58.

38 Welfare MR, Aitkin M, Bassendine MF, Daly AK. Detailed modelling of caffeine metabolism and examination of the CYP1A2 gene: lack of a polymorphism in CYP1A2 in Caucasians. Pharmacogenetics 1999; 9: 367-75.

39 Perry PJ, Bever KA, Arndt S, Combs MD. Relationship between patient variables and plasma clozapine concentrations: a dosing nomogram. Biol Psychiatry 1998; 44: 733-8.

40 Ozdemir V, Kalow W, Posner P, Collins EJ, Kennedy JL, Tang BK, et al. CYP1A2 activity as measured by a caffeine test predicts clozapine and active metabolite steady-state concentrationin patients with schizophrenia. J Clin Psychopharmacol 2001; 21: 398-407. 